\title{
Extranodal NK/T-Cell Lymphoma: Toward the Identification of Clinical Molecular Targets
}

\author{
Christian Schmitt, ${ }^{1,2,3}$ Nouhoum Sako, ${ }^{1,2}$ Martine Bagot, ${ }^{1,2}$ Yenlin Huang, ${ }^{4}$ \\ Philippe Gaulard, 5, 6,7 and Armand Bensussan ${ }^{1,2}$ \\ ${ }^{1}$ INSERM U976, 75010 Paris, France \\ ${ }^{2}$ Faculté des Sciences, Université Paris Diderot, 75013 Paris, France \\ ${ }^{3}$ Immunologie, Oncologie, et Dermatologie, INSERM U976, Hôpital Saint-Louis, \\ 1 avenue Claude Vellefaux, Pavillon Bazin, 75475 Paris Cedex 10, France \\ ${ }^{4}$ Department of Anatomic Pathology, Chang Gung Memorial Hospital, Gueishan 33305, Taiwan \\ ${ }^{5}$ INSERM U955, 94010 Créteil, France \\ ${ }^{6}$ Faculté de Médecine, Université Paris-Est Créteil, 94010 Créteil, France \\ ${ }^{7}$ Groupe Henri-Mondor, Département de Pathologie, AP-HP, 94010 Créteil, France
}

Correspondence should be addressed to Christian Schmitt, christian.schmitt@inserm.fr

Received 30 December 2010; Accepted 24 February 2011

Academic Editor: John E. Coligan

Copyright ( $) 2011$ Christian Schmitt et al. This is an open access article distributed under the Creative Commons Attribution License, which permits unrestricted use, distribution, and reproduction in any medium, provided the original work is properly cited.

\begin{abstract}
Extranodal natural killer (NK)/T-cell lymphoma of nasal type (NKTCL) is a malignant disorder of cytotoxic lymphocytes of NK or more rarely T cells associated with clonal Epstein-Barr virus infection. Extranodal NKTCL is rare in Western countries, but in Asia and Central and South America it can account for up to $10 \%$ of non-Hodgkin's lymphomas. It is an aggressive neoplasm with very poor prognosis. Although the pathogenesis of extranodal NKTCL remains poorly understood, some insights have been gained in the recent years, especially from genome-wide studies. Based on our own experience and knowledge of the literature, we here review some of the genomic and functional pathway alterations observed in NKTCL that could provide a rationale for the development of innovative therapeutic strategies.
\end{abstract}

\section{Introduction}

The term extranodal natural killer/T-cell lymphoma (NKTCL) refers to a group of clonal proliferations of cytotoxic lymphocytes of natural killer (NK) or, more rarely, T-cell types, with peculiar clinicopathologic features, arising mainly as tumors or destructive lesions in the nasal cavity, maxillary sinuses, or palate [1]. More rarely, extranodal NKTCL may present in other extranodal sites such as skin, testis, lung, or gastrointestinal tract and tend to have a more adverse clinical outcome [2-4]. This is particularly true when one defines nonnasal cases as extra upper aerodigestive tract cases as in the study of Lee et al. which reports survival rates of $20 \%$ versus $54 \%$ for the patients with nasal and upper airway region localizations [4]. However, as noted by several authors, many nonnasal NKTCL might represent disseminated nasal NKTCL, knowing that such dissemination can occur early in the clinical course of the disease and toward sites that are localizations where nasal NKTCL will metastasize to. Besides the more adverse clinical features, for which the underlying mechanisms need to be defined, there are no significant differences in age, gender, ethnicity, bone marrow involvement, hemophagocytosis, or immunophenotypic profiles between nasal and nonnasal NKTCL. Very rare cases with primary lymph node involvement have also been described [5]. Extranodal NKTCL shows a wide cytological spectrum and is characterized by frequent features of angioinvasion and angiocentrism, which often result in coagulative necrosis. Typically, tumor cells express cytoplasmic CD3e, CD2, CD56, lack CD5, CD4, and CD8, and have an activated cytotoxic immunophenotype with expression of perforin, 
granzyme B, and TiA1. The cell of origin of these tumors has been debated. Indeed, the identification of CD3 expression in tumor cells in necrotic lesions has led to the terminology of angiocentric T-cell lymphoma [6-8] adapted in the REAL classification [9], before it was demonstrated that tumor cells express several chains of the CD3 complex in the cytoplasm, but lack T-cell receptors (TCR) and have TCR genes in a germline configuration, consistent with an NK-cell origin $[10,11]$. If the majority of NKTCL are likely to originate from mature NK cells, a small proportion of cases, with expression of $\gamma \delta$ or $\alpha \beta$ TCR appear to derive from cytotoxic T lymphocytes, reflected in the "NK/T" cell terminology.

Extranodal NKTCL represents the major group of mature NK cell neoplasms in the recently revised WHO classification of hematolymphoid tumors, which also include the aggressive NK cell leukemia (ANKL) and a provisional group of chronic NK-cell lymphoproliferative disorder of uncertain malignant potential, most likely related to T-cell large granular lymphomas [1]. Importantly, both NKTCL and ANKL are Epstein-Barr virus- (EBV-) associated neoplasms as the virus is found in their tumor cells $[12,13]$. Although the precise role of the virus in the etiology of the disease is poorly understood, the study of EBV gene polymorphism has shown that tumor cells are clonally infected as opposed to normal nasal tissues $[14,15]$. Circulating EBV viral load is an important prognostic factor, and plasma EBV DNA levels can also be used for disease monitoring [16]. In this respect, the incidence of NKTCL parallels the geographic distribution of EBV infection with prevalence in the Asian and Central and South American populations, where it can account for up to $10 \%$ of non-Hodgkin's lymphomas [17-19].

Despite a localized presentation in most patients, extranodal NKTCL is an aggressive disease with poor prognosis. The 5-year survival rate is less than $50 \%$. In the absence of effective treatment, the median survival for advancedstage disease is only 6-12 months [19-22]. The retrospective International Peripheral T-cell Lymphoma project recently reported a median overall survival of 7.8 months for NKTCL, corresponding to the poorest survival among all T-cell lymphoma entities [2]. Therefore, despite progress with combined field radiotherapy and chemotherapy, autologous bone marrow transplantation and the promising effect of Lasparaginase treatment in relapsed cases $[16,23]$, NKTCL remains difficult to cure, and the need for alternative therapeutic strategies has prompted researchers to explore oncogenic pathways involved, to provide new molecular targets. This review will focus on the these potential molecular pathways that have been implicated in the physiopathology of NKTCL, in particular through the lights shed by several recently reported genome-wide profiling studies [24-30].

\section{EBV Infection and Viral Protein Expression}

Several lines of evidence point at EBV as a major player in the pathogenesis of NKTCL. First of all, when dealing with an EBV-associated malignancy, one can think of NKTCL as a potentially highly immunogenic lymphoma that could benefit from cellular immunotherapies targeting the viral antigens as in posttransplant B-cell lymphoproliferative disorders [31]. These B-cell proliferations, like the in vitro infected Bcell-derived lymphoblastoid cell line (LCL), express the full spectrum of EBV latent proteins (latency III). The latent phase consists of the maintenance of the EBV genome as a circular episome that is replicated by the cellular DNA polymerase. Latent type III infection is associated with the expression of six EBV-encoded nuclear antigens (EBNA): EBNA1, EBNA2, EBNA2A, EBNA3B, EBNA3C, and EBNA leader protein (EBNA-LP); three cell surface proteins: latent membrane protein (LMP) 1, LMP2A, and LMP2B; two EBVencoded RNAs: EBER1 and EBER2. Among these proteins, the EBNA3s are believed to be particularly immunogenic for the generation of specific cytotoxic T lymphocytes (CTL). However, similarly to Hodgkin's lymphoma, NKTCL is believed to be in latency II, with a more restricted pattern of viral proteins expressed, limited to EBNA1, LMP1, and LMP2 [32]. EBNA1 is required for the maintenance of viral episomes and expressed in all latency phases. LMP1 is the main transforming protein of EBV. It is essential for EBV-induced B-cell transformation in vitro, acting like a constitutively activated tumor necrosis factor receptor family member, activating NF- $\kappa$ B pathway and promoting cell survival. LMP2A and LMP2B are generated by alternative splicing and, although not essential for B-cell transformation, can also promote proliferation and survival. However, LMP2 has only been demonstrated at the transcriptional level in NKTCL. LMP1 expression is also variable, and when expressed it is often limited to a subpopulation of tumor cells, possibly under the pressure of host immune surveillance. Despite these challenging conditions, attempts have been made to generate in vitro LMP2-specific CTL using antigenpresenting cells overexpressing LMP2, to treat Hodgkin's lymphoma and NKTCL patients [33]. The cytotoxic activity of these LMP2-specific CTL have been confirmed against NKTCL-derived cell line, which led to the discovery of a unique alternative transcript of LMP2 (LMP2-TR) that uses a new promoter located in the terminal repeat region of the episomal EBV, not expressed in B-LCL [34]. LMP2TR may constitute a promising target for adoptive cellular immunotherapy in NKTCL.

EBV is a B-lymphotropic virus, infecting B cells through interaction with its cellular receptor CD21, and establishing a lifelong infection in more than $90 \%$ of the human adult population. Although the precise mechanism involved in NK- or T-cell infection is unknown, passive acquisition of CD21 in the NK/T-cells by membrane fragment exchange during cytotoxic interaction, a process known as trogocytosis, has been proposed $[35,36]$. Chronic active EBV infection (CAEBV) was first recognized as severe illness related to chronic or persistent EBV infection [37, 38]. CAEBV is a life-threatening disease characterized by infectious mononucleosis-like symptoms with virus-associated hemophagocytic syndrome. Like in NKTCL, clonal proliferations of EBV-infected NK- or T-cells are seen in patients with CAEBV, making this disease more likely a chronic lymphoproliferative disorder or a premalignant stage as some CAEBV patients can actually develop subsequent NKTCL [39]. Whether the EBV-host interaction may play a role 
in the development of NKTCL is still a pending question. Performing comparison of EBV and cellular gene expression profiling in NKTCL- and CAEBV-derived cell lines, Zhang et al. reported the transcription of lytic phase genes such as BZLF1, BARF1,BFLF2, and BDLF3, despite the absence of virion DNA production and cellular gene signature implicating mainly cell cycle and apoptosis-related genes such as TNFRSF10D, CDK2, Hsp90, IL12A, and PDCD4 [30]. Beside the expression of latency II genes, expression in NK/T-cell lines, of lytic phase genes (such as BZLF1) capable to interact with multiple cell cycle control proteins is intriguing. EBV may exert oncogenic effects through the production of cytokines such as Interleukin 9 (IL-9) [26] and IL-10 [40]. EBV-induced production of IP10/MIP2 chemokines may contribute to vascular damage and necrosis [41]. Obviously, the way viral gene products interact with cellular host metabolism needs to be clarified and may lead to novel targets for therapies.

\section{NK Cell Antigens and Cytotoxic Activity}

Irrespective of their $\mathrm{NK}, \alpha \beta$-, or $\gamma \delta$-T-cell lineage, NKTCL is a lymphoma of activated cytotoxic lymphocytes as evidenced by the expression of cytotoxic molecules such as perforin, granzyme B, and TIA1 [42]. Whereas T cells use their TCR for antigen recognition and control of their specific cytotoxic activity, NK cells use MHC class I-specific NK cell receptors (NKR) for this purpose [43]. NK cells are part of the innate immune system involved in the immunosurveillance [44]. Engagement of inhibitory NKR by self-MHC class I on potential target cells inhibits cytotoxicity whereas loss of MHC class I expression due to transformation or viral infection, results in a loss of this inhibition. There are two types of NKR: the killer immunoglobulin-like receptors (KIR) and the killer cell lectin-like receptors (KLR or CD94/NKG2 complexes). KLR as well as other NK cell markers like CD56 can also be expressed by $\alpha \beta$ - or $\gamma \delta$-cytotoxic T cells. The KIR locus located on human chromosome 19, exhibits a substantial haplotypic and allelic diversity $[45,46]$. The KIR haplotypes have been separated into two groups, containing from 7 to 12 genes [47]. The expression of KIR at the cell surface is clonally distributed, meaning that a clonal NK/T-cell population is expected to express a restricted KIR repertoire as shown in NKTCL [48-50]. Another example of the interest of KIR in malignant lymphomas is given by KIR3DL2 that proved to be a valuable tool in the diagnosis, and perhaps a clinical target, of Sézary syndrome, a cutaneous $\mathrm{CD} 4^{+} \mathrm{T}$-cell lymphoma with systemic dissemination [51-53]. KLR are C-type lectin receptors that precede the expression of KIR during NK cell development. They consist of molecules of the NKG2 family such as NKG2A (KLRC1), NKG2C (KLRC2), or NKG2E (KLRC3), form heterodimer with earlier expressed CD94 at the surface of maturing NK cells [54]. In addition to immature NK cells, a subset of mature NK cells also lack CD94/NKG2 expression as a consequence of the clonal diversification phenomenon. Interestingly, expression of CD94 has been associated with a better prognosis in NKTCL [55]. Although NK activity was identified from the initial description of ANKL $[56,57]$, the cytotoxic activity of the NKTCL tumor cells has not been particularly investigated due to the difficulty to isolate these cells.

\section{Survival/Apoptosis}

The mechanisms by which NKTCL cells survive and escape immune surveillance are unknown. One way for an NKTCL tumor cell to escape anti-EBV immunity is to lower its expression of EBV antigens, particularly LMPs proteins. It is also known that cytotoxic effector cells have a proper way to resist to their own cytotoxic agents [58]. The main cytotoxic pathway used by NK and CTL involves the release from specialized granules of cytotoxic molecules including perforin and granzymes, triggering a process leading to DNA fragmentation and apoptosis. In particular, granzyme B (GZMB), by activating directly or indirectly caspase-3, leads to rapid execution of apoptosis. SERPINB9 (also known as PI9) is a GZMB-specific serine protease inhibitor that can protect effector cells from their own cytotoxic activity. It has been shown that SERPINB9 can make hepatocytes resistant to lysis by CTL or NK cells [59] and may participate to the mechanism for tumor escape in NKTCL [60]. Interestingly, its loss of expression was described as a poor prognostic factor in a study of 48 NKTCL patients [20].

Although it seems that tumor cells are resistant to the killing via GZMB, NKTCL are frequently associated with zonal tumor cell death, tissue necrosis, and vascular damage. The production of chemokines like CXCL9 (Mig) and CXCL10 (IP10), in response to interferon $\gamma$ released by activated tumor cells, may contribute to the observed damages [41, 61]. Although Fas (CD95) as well as Fas ligand (FASL/CD95L) are frequently well expressed in NKTCL cells, mutations of the FAS gene are observed in 50 to $60 \%$ of cases, most likely contributing to resistance to apoptosis [62]. Indeed, most of the FAS gene mutations are frameshift mutations arising in the death domain, leading to mutated proteins on the cell surface, unable to transduce the apoptotic signal [63]. High expression of FASL is also a way for tumor cells to evade immune response by deleting Fasexpressing infiltrating $\mathrm{T}$ cells. In line with these observations, NKTCL has long been known as resistant to combination chemotherapy. This resistance is also complicated by the high expression of p-glycoprotein, encoded by multidrug resistance gene 1 (MDR1) that acts as an energy-dependent efflux pump for various drugs [64].

Very recently, survivin (BIRC5), an inhibitor of apoptosis frequently involved in tumor oncogenesis, was found overexpressed in NKTCL [28]. The authors proposed that survivin could be a useful therapeutic target in NKTCL. In in vitro studies, they showed that Terameprocol, a survivin inhibitor, could downregulate survivin expression and induced apoptosis in NK cell lines.

\section{Cytogenetic Analysis}

Lymphomas arise from clonal expansion of transformed lymphoid cells through the accumulation of genetic lesions. 
Currently, no genetic abnormalities specific for NKTCL have been identified. Cytogenetic analyses of NKTCL are difficult because of necrosis, small-size samples, and contamination by inflammatory reactive cells. Despite these difficulties, several studies using comparative genomic hybridization (CGH) and loss of heterozygosity (LOH) techniques have been reported [65-70]. Altogether, these studies identified gains at chromosomes $1 \mathrm{p}, 6 \mathrm{p}, 11 \mathrm{q}, 12 \mathrm{q}, 17 \mathrm{q}, 20 \mathrm{q}, \mathrm{Xp}$, and losses at $6 \mathrm{q}, 11 \mathrm{q} 13 \mathrm{q}$, and $17 \mathrm{p}$. In particular, the most frequent deletion was observed at 6q21-25 in all but one study. Sun et al. tried to define a minimal tumor suppressor gene-containing region involved in del6q25 and identified a $2.6 \mathrm{Mb}$ interval located between TIAM2 and SNX9 genes [69]. More recently, progress in the DNA chip technology allowed the development of genome-wide arraybased CGH (aCGH) giving access to better resolutions. The first report, from Nakashima et al., performed with homemade arrays with $1.35 \mathrm{Mb}$ resolution, studied 10 of ANKL and 17 NKTCL cases and reported differences in their genomic alteration patterns (see Table 1) [27]. Loss of $7 \mathrm{p}$ $(40 \%)$ and $17 \mathrm{p} 13.1(40 \%)$ and gain of $1 \mathrm{q}(43 \%)$ occurred more frequently in ANKL whereas loss of $6 \mathrm{q}$ did not. On the other hand, loss of 6q21-q22.1 (35\%), 6q22.33-q23.2 (47\%), 6q25.3 (29\%), and 6q26-q27 (35\%) were more frequent in NKTCL. Other alterations in NKTCL compared to ANKL include gain in $2 \mathrm{q}$ and loss of $1 \mathrm{p} 36.23-\mathrm{p} 36.33,2 \mathrm{p} 16.1-$ $\mathrm{p} 16.3,4 \mathrm{q} 12,4 \mathrm{q} 31.3-\mathrm{q} 32.1,5 \mathrm{p} 14.1-\mathrm{p} 14.3,5 \mathrm{q} 34-\mathrm{q} 35.3$, and 11q22.3-q23.3. Two recent studies extended these findings by comparing submegabase resolution aCGH and gene expression profiling on NK cell lines as well as NKTCL tumor samples $[24,71]$. These two studies emphasize recurrent genomic abnormalities observed in about half of the patients: gain of 1q21-q44, and losses of 17p11.2-p13.3 and 6q21. In particular, this region of del6q21 contains four candidate tumor suppressor genes, PRDM1, ATG5, AIM1, and HACE1, which decreased expression was confirmed at the RNA level. In their study, Iqbal et al. found mutations and methylation in PRDM1, ATG5, and AIM1 in NKTCL cell lines [71]. ATG5 is part of the autophagy pathway implicated both in apoptosis and in the maintenance of energy homeostasis during starvation [72]. The role of autophagy in oncogenesis is, however, the object of controversy [73]. AIM1 which stands for "absent in melanoma-1" is still poorly characterized but was reported as a good tumor suppressor gene candidate in malignant melanoma, exerting its effects through interactions with the cytoskeleton [74]. PRDM1 is a transcriptional regulator, initially described as a suppressor of beta-interferon gene expression, that is associated with the terminal differentiation of B lymphocytes and T-cell homeostasis and function $[75,76]$. It was found mutated in some diffuse large B-cell lymphomas, and it was concluded that its inactivation contributes to lymphomagenesis by blocking differentiation of postgerminal center B cells to plasma cells [77]. Loss-of-function due to mutations as well as transcriptional inhibition by DNA methylation observed in NK cell lines, suggest a tumor suppressor role of PRDM1 in NKTCL [71].

HACE1 is another candidate tumor suppressor gene [24, 90]. Characterization of the chromosome 6q21 breakpoint, frequently involved in sporadic Wilm's tumors, led to the identification of the HACE1 gene [91]. It encodes a member of the HECT family of E3 ubiquitin ligases that tag specific target proteins to degradation by the proteasome or to control their subcellular localization [85]. Because of their involvement in controlling crucial signaling pathways, E3 ubiquitin ligases appear as important regulators of cancer development and therapy. It is downregulated in multiple human cancers and maps to a prominent tumor-suppressor region of LOH in many tumors including lymphomas. $\mathrm{HACE}^{-/-}$mice are also prone to spontaneous development of various types of cancers [90]. Our integrated genomic and transcriptomic analysis of NKTCL tumor samples and cell lines has shown downexpression of HACE1 gene irrespective of the presence of del6q21 alteration [24]. In addition, we could show by methylation-specific PCR and product sequencing (data not shown) that hypermethylation of CpG177 island located directly upstream of HACE1 locus, is responsible of the silencing of the diploid gene or the remaining allele. Although the mechanism of antitumor action of HACE1 is still unclear, it has been shown that overexpression of HACE1 in the 293T cell line inhibits cell proliferation via the degradation of phosphorylated cyclin D1 [90]. This suggests a possible mechanism for HACE1 to regulate cell cycle exit by reducing cyclin D1 levels. Existence of other HACE1 targets is possible as suggested by the recent identification of retinoic acid receptor beta 3 as an HACE1 interacting protein [92].

\section{Cell Signaling Pathways}

Although the anomaly of signaling pathways involved in the pathogenesis of NKTCL is not deciphered, progress has been made in the recent years that will help to define candidate therapeutic targets.

6.1. Jak-Stat Pathways. Signal transducers and activators of transcription, STATs, are transcription factors activated in response to cytokines or growth factors [80]. The activation of STAT signaling pathways requires tyrosine phosphorylation of the STAT proteins that results from their association with growth factor receptors having intrinsic tyrosine kinase activity, or through recruitment of members of the Janus kinase (JAK) family to activated surface receptors. A direct link between STAT signaling and oncogenesis was established when it was shown that constitutive STAT activation by oncogenic tyrosine kinases from $\mathrm{Src}$ or $\mathrm{Abl}$ families, directly participates to cell transformation [93, 94]. Accordingly, a large number of tumors, both in primary cells as well as in tumor-derived cell lines, display constitutive activation of STAT factors. In particular, STAT3 role in oncogenesis is well documented in anaplastic large cell lymphoma where STAT3 activation was shown to provide growth advantage and resistance to apoptosis of $\mathrm{ALK}^{+}$tumor cells $[81,95,96]$. In NKTCL, STAT3 was found constitutively activated, by phosphorylation on Y705, and localized in the nucleus in $90 \%$ of the patients studied $[24,82]$. Inhibition of endogenous 
TABLE 1: Recurrent genomic alterations in extranodal NKTCL from published data.

\begin{tabular}{|c|c|c|c|c|c|c|c|}
\hline \multirow{2}{*}{$\begin{array}{l}\text { Chromosomal } \\
\text { location }\end{array}$} & \multicolumn{7}{|c|}{ Frequency (\%) } \\
\hline & $\begin{array}{c}\text { Huang et al. } \\
2010[24]\end{array}$ & $\begin{array}{c}\text { Iqbal et al. } 2009 \\
{[71]}\end{array}$ & $\begin{array}{l}\text { Nakashim et al. } \\
2005 \text { [27] }\end{array}$ & $\begin{array}{c}\text { Yoon and Ko } \\
2003[70]\end{array}$ & $\begin{array}{c}\text { Ko et al. } 2001 \\
{[66]}\end{array}$ & $\begin{array}{c}\text { Siu et al. } 2000 \\
{[67]}\end{array}$ & $\begin{array}{c}\text { Siu et al. } 1999 \\
{[68]}\end{array}$ \\
\hline \multicolumn{8}{|l|}{ Gains } \\
\hline $1 \mathrm{q} 21-\mathrm{q} 44$ & 50 & 50 & 50 & & & & 20 \\
\hline $2 q 13-q 14$ & & 26 & 24 & & 29 & & \\
\hline $2 \mathrm{q} 31.1-\mathrm{q} 32.2$ & & 20 & 24 & & 43 & & \\
\hline $6 \mathrm{p} 25-\mathrm{p} 11.1$ & 40 & 25 & 40 & & & & \\
\hline $7 q 11.2-q 34$ & 50 & 32 & 24 & & & & 20 \\
\hline $7 q 35-q 36$ & 60 & 40 & & & & & 40 \\
\hline $17 q 21.1$ & 20 & 50 & & & 29 & & 40 \\
\hline 20pter-qter & 30 & 45 & & & & & 50 \\
\hline \multicolumn{8}{|l|}{ Losses } \\
\hline $6 q 16-q 25$ & 40 & 50 & 38 & 80 & & 49 & 25 \\
\hline $11 q 23.1$ & 30 & 13 & 29 & & & 31 & 20 \\
\hline $11 q 24-q 25$ & 40 & 25 & & & & & 20 \\
\hline $13 q 14.11$ & 30 & 27 & 25 & & & 14 & 60 \\
\hline $17 \mathrm{p} 13.3$ & 40 & 45 & 40 & & 43 & 40 & 20 \\
\hline
\end{tabular}

TABLe 2: Potential Therapeutic targets for extranodal NKTCL.

\begin{tabular}{|c|c|c|c|c|}
\hline Pathways & Genes & Expression & Chromosomal location & References \\
\hline EBV & LMP2-TR & Up & N.A. & {$[32]$} \\
\hline \multicolumn{5}{|c|}{ Survival/apoptosis } \\
\hline & CCND3 & Down & $6 \mathrm{p} 21.1$ & [69] \\
\hline & SERPINB9 & Down & $6 \mathrm{p} 25$ & {$[19,57]$} \\
\hline & FASL & $\mathrm{Up}$ & $1 q 23$ & {$[60,78]$} \\
\hline & TNFAIP3 & Down & $6 \mathrm{q} 23$ & {$[69,79]$} \\
\hline \multicolumn{5}{|c|}{ Cell signaling pathways } \\
\hline \multirow[t]{3}{*}{ JAK-STAT } & STAT3 & Up & $17 q 21$ & {$[80,81]$} \\
\hline & JAK2 & Up & $9 \mathrm{p} 24$ & {$[69,81]$} \\
\hline & IL10 & Up & $1 q 31-q 32$ & {$[81,82]$} \\
\hline AKT & $\mathrm{AKT} 1 / 2 / 3$ & Up & $14 q 32.3 / 19 q 13.1 / 1 q 44$ & {$[69,70]$} \\
\hline NOTCH & NOTCH1 & $\mathrm{Up}$ & $9 q 34.3$ & {$[24,83]$} \\
\hline WNT & $\beta$-CATENIN & Up & $3 \mathrm{p} 22-\mathrm{p} 21.3$ & {$[24,69]$} \\
\hline \multirow[t]{2}{*}{ PDGF } & PDGFRA & Up & $4 \mathrm{q} 11-\mathrm{q} 13$ & {$[69]$} \\
\hline & PDGFA/B & Up & $7 \mathrm{p} 22 / 22 \mathrm{q} 12.3-\mathrm{q} 13.1$ & {$[69]$} \\
\hline \multicolumn{5}{|c|}{ Angiogenesis } \\
\hline & HGFR (MET) & Up & $7 q 31$ & {$[78]$} \\
\hline & VEGFR2 & Up & $4 \mathrm{q} 12$ & {$[24,69]$} \\
\hline & VEGFA & Up & $6 \mathrm{q} 12$ & {$[24,69]$} \\
\hline & HIF $1 \alpha$ & Up & $14 \mathrm{q} 21-\mathrm{q} 24$ & {$[24,70,84]$} \\
\hline \multicolumn{5}{|c|}{ Tumor suppressor genes } \\
\hline \multirow[t]{4}{*}{ del6q21 } & PRDM1 & Down & $6 \mathrm{q} 21-\mathrm{q} 22.1$ & {$[70]$} \\
\hline & ATG5 & Down & $6 q 21$ & {$[70]$} \\
\hline & AIM1 & Down & $6 \mathrm{q} 21$ & {$[70]$} \\
\hline & HACE1 & Down & $6 q 21$ & {$[69,85]$} \\
\hline \multicolumn{5}{|c|}{ Other pathways/genes } \\
\hline & TP53 & Down & $17 \mathrm{p} 13.1$ & {$[86,87]$} \\
\hline & TP73 & Down & $1 \mathrm{p} 36.3$ & {$[88]$} \\
\hline & AURKA & Up & $20 \mathrm{q} 13$ & {$[24,89]$} \\
\hline
\end{tabular}

N.A.: not applicable 
activated STAT3 in NKTCL-derived cell lines, using transducible dominant-negative STAT3, leads to growth arrest and apoptosis inhibition [82]. These results are consistent with the known targets of STAT3 transcriptional activities that include survivin, cyclin D1, BCL- $\mathrm{X}_{\mathrm{L}}$, cMYC, vascular growth factor (VEGF), IL-10, and IL-6 [97]. Importantly, STAT3 activation promotes the production of immunosuppressive factors not only by the tumor cells but also by cells of the microenvironment through mediators such as VEGF or IL-10, activating STAT3 in these bystander cells that in turn restrains antitumor immune response [84, 98]. Targeting STAT3 and/or JAK2 for cancer immunotherapy may therefore be promising.

6.2. Angiogenesis Pathway. NKTCL is characterized by angiocentric growth and vascular damages with invasion of vessel walls by lymphoma cells. In this context, a search for genes associated with angiogenesis seems essential. The recent gene expression profiling studies have recorded overexpression of genes related to angiogenesis in NKTCL as compared to other peripheral $\mathrm{T}$ cell lymphomas or normal NK cells $[24,25]$. Among those, one finds VEGFA an important downstream product of STAT3, and its receptor VERGFR2 $(K D R)$, both genes are upregulated, a finding confirmed by the immunohistochemical detection of $\operatorname{VEGF} \alpha$ and its receptor in tumor cells. As mentioned previously, the production of VEGF by tumor cells not only may be used for autocrine growth and survival but also to maintain an immunosuppressive microenvironment by activating the STAT3 pathway. Vascular destruction observed in nasal type NKTCL results in hypoxia and the transcription activation of $\operatorname{HIF} \alpha$ (hypoxia-inducible factor 1), a transcriptional activator that stimulates the expression of a number of genes implicated in angiogenesis (including VEGFA and VEGFR2) and in oxygen homeostasis [78]. Also observed is the upregulation of another important gene in angiogenesis, the $c-M E T$ proto-oncogene that encodes the tyrosine kinase cell surface receptor for the hepatocyte growth factor (HGF). HGF is a pleiotropic cytokine acting as an antiapoptotic, a promigratory, and a proliferating factor for a number of tissues. Interestingly, MET may be implicated in the prevention of FAS-mediated apoptosis of the FAS-expressing NKTCL tumors cells. This relies on the YLGA aminoacid motif located near the N-terminal region of MET that specifically binds to the extracellular portion of FAS and acts as a FASL antagonist and inhibitor of FAS trimerization [99].

6.3. PDGFR Pathway. The platelet-derived growth factor receptor- (PDGFR-) signaling pathway was also found activated in NKTCL cells [24]. The overexpression of PDGFRA mRNA in NKTCL compared to normal NK cells in microarray data is confirmed by the expression of PDGFR $\alpha$ and its phosphorylated form in primary tumors, shown by immunohistochemistry. Neither genomic abnormalities in the PDGFRA locus in 4q11-q13 nor mutations in the coding sequence or anomalies in the promoter region could be evidenced. The mechanism responsible for the activation of the PDGFR remains therefore unclear, but like in PTCL NOS (PTCL not otherwise specified) [83], an autocrine feedback loop may explain the presence of the phosphorylated PDGFR $\alpha$ observed in all the 13 NKTCL cases examined. The potential of PDGF signaling pathway in NKTCL cell proliferation is illustrated by the dramatic dose-dependent inhibition of MEC04 cell growth observed in cultures performed the presence of imatinib mesylate [24].

6.4. Other Signaling Pathways. Pathways enriched in the gene signature of NKTCL include those of the NOTCH, WNT, and $\mathrm{NF} \kappa \mathrm{B}$ signaling pathways $[24,25,30]$. However, and particularly in this tumor where necrosis is associated with strong infiltration of mesenchymal and lymphoid cells, it is sometimes difficult to attribute the differentially expressed genes to the tumor cells rather than to the microenvironment cells. In addition, differential expression needs confirmation in terms of biochemical activation. In particular, activation of the WNT pathway, comprising genes involved during the development and in oncogenesis, can be evidenced by the nuclear translocation of $\beta$-catenin. However, no nuclear expression of $\beta$-catenin could be observed in the neoplastic cells in our study by Huang et al. [24], making the targeting of WNT pathway of poor interest for therapeutic purpose. On the other hand, activation of the NOTCH pathway has been validated by Iqbal et al. [25]. NOTCH signals regulate development and differentiation of adult selfrenewing cells, including T cells. Gamma-secretase is a critical component of the NOTCH signal transduction pathway [100]. Showing that inhibitors of $\gamma$-secretase can block the proliferation and survival of NK cell lines in vitro, was used for validation of NOTCH implication. Interestingly, PTEN is a tumor suppressor gene encoding a lipid phosphatase that antagonizes the activation of the PI3K/AKT pathway. NOTCH1 controls a transcriptional network that leads to activation of PI3K/AKT signaling and downmodulation of PTEN expression. NOTCH signaling and PI3K/AKT pathway act synergistically to maintain oncogenic activity in T-cell acute lymphoblastic leukemia [101]. PI3K/AKT was found activated in microarray analysis of NKTCL, and nuclear expression of phosphorylated-AKT was found in the nucleus of most NKTCL samples. NF $\kappa B$ is a master regulator that controls the expression of a number of genes. Because EBV is known to activate $\mathrm{NF} \kappa \mathrm{B}$ through LMP-1 and/or TRAF signaling [79], upregulation of $\mathrm{NF} \kappa \mathrm{B}$ pathway in NKTCL versus normal NK cells is not unexpected. Signaling by the transcription factor $\mathrm{NF} \kappa \mathrm{B}$ involves its release from its inhibitor $\mathrm{I} \kappa \mathrm{B}$, followed by its translocation into the nucleus. Nuclear detection by immunohistochemistry of RelA, the protein constituting the most abundant form of $\mathrm{NF} \kappa \mathrm{B}$, further supports the activation of this pathway in NKTCL. Interestingly, the tumor necrosis factor- $\alpha$-induced protein gene, TNFAIP3, an inhibitor of NF $\kappa$ B activity, was also found downregulated in NKTCL. This gene is located in $6 \mathrm{q} 23$, the region of recurrent loss discussed above, and loss of function of TNFAIP3 have also been reported in Hodgkin's lymphoma and other B-cell lymphomas [86, 102]. Surprisingly, the $\mathrm{NF} \kappa \mathrm{B}$ pathway genes were not enriched in the NKTCL gene signature in the recent study of Iqbal et al. [25]. Questions 
remain, therefore, to clarify the value of this pathway to identify therapeutic targets for NKTCL.

\section{Other Pathways and Candidate Target Genes}

Many other genes are found differentially expressed or targets for mutations or methylations in NKTCL when compared to normal NK cells (see Table 2). The list includes a number of oncogenes or tumor suppressor genes associated with many other tumors. TP53 is a well-known tumor suppressor gene that causes cells with damaged DNA to arrest at G1 phase of the cell cycle. TP53 is mutated in many cancers, and the frequency of NKTCL-mutated cases varies from 20 to $60 \%$. TP53 mutated cases have been associated with more advanced cases, suggesting that TP53 mutation represents a secondary oncogenic event rather than a triggering mechanism for the development of NKTCL [87]. Nevertheless, TP53 is found underexpressed in NKTCL transcriptomic analyses, and the observation that EBNA1 promotes TP53 degradation [88] has to be considered to make TP53 pathway a possible target for NKTCL therapy. TP73 belongs to the family of p53-related proteins, which is involved in cell cycle arrest and apoptosis. Methylation of TP73 has been reported in 94\% of NKTCL and has been proposed as a biomarker to detect NKTCL involvement and metastasis [103]. MYC family genes encode multifunctional nuclear phosphoproteins that function as transcription factors to regulate expression of genes involved in cell cycle progression such as CCNA2, CDKN1A, and $C D K N 2 B$. Although frequent in hematopoietic and solid tumors, mutations, amplification, and translocation of this oncogene have not been found in NKTCL. However, MYC expression appears to be upregulated in the gene signature of NKTCL.

Adhesion and cell-to-cell interaction molecules may play an important role in tumor growth and homing in view of the particular tropism of NKTCL for extranodal sites. The molecular signatures reported for NKTCL in comparison with normal NK cells include overexpression of genes of the cadherin family (CDH1), of the integrin family (VCAM1, ITGA7, ITGA9, and ITGB4), and of the sialoadhesin family (SIGLEC1, SIGLEC 9, SIGLEC11, and SIGLECP3). Protocadherin 15 (PCDH15) was shown to be expressed ectopically by NKTCL cells and NKTCL-derived cell lines [104], and confirmed in gene expression profiling [24]. PCDH15, normally absent in the hematopoietic tissues, may be used by NK tumor cells to escape immunosurveillance, like CDH1 recognized by the KLRG1 inhibitory NK receptor [105].

Recently, AURKA, the Aurora kinase A or ST6, was reported as another potential target for NKTCL [25]. AURKA is a mitotic centrosomal protein kinase that controls chromosome segregation during mitosis $[89,106]$. AURKA is activated by phosphorylation and could be detected in all human NK cell lines tested, validating its transcriptomic overexpression observed in NKTCL versus normal NK cells. In many other cancers, AURKA upregulation results in a phenotype characteristic of loss-of-function mutations of
TP53. Katayama et al. showed that TP53 is phosphorylated by AURKA, inducing its ubiquitination by MDM2 and its proteasomal degradation [107]. Thus, AURKA is a key regulator of TP53 pathway, and the AURKA inhibitor MK8751 induced cell cycle arrest and apoptosis in human cell lines, including NKTCL-derived cell lines [25]. AURKA represent, therefore, an interesting potential therapeutic target for NKTCL.

\section{Conclusion}

ANKL and NKTCL are EBV-associated malignancies of cytotoxic lymphocytes with poor prognosis and lack of efficient therapies. Their aggressive behavior and poor response to chemotherapy make of paramount importance the search for novel therapeutic targets in the treatment of these lymphomas. NKTCL is a rare disease although more frequent in Asia and South America, and the insufficient supply of tumor samples limits the development of an intense research in that field. In this context, malignant NK/T-cell lines represent valuable tools to study these pathologies [108]. Genome-wide studies have generated new data improving our understanding of the disease. Indeed, candidate tumor suppressor genes, such as PRDMI, ATG5, AIM1, or HACE1, which can be inactivated by deletion and by methylation, have been identified. The molecular signature of NKTCL is distinct from that of other T-cell lymphoma subtypes. Compared to normal NK cells, NKTCL is characterized by activation of several pathways-PDGFRA, VEGFR, AKT, JAK-STAT, and NOTCH — which might represent targets for novel therapeutic options. Before settingup clinical trials, extensive experimental validation of the potential target will have to be undertaken using the available models. In this respect, the study of tumor engrafting and development in immunocompromised mice injected with NKTCL cell lines or tumor fragments $[82,109]$ can be valuable models to test new potential drugs and their in vivo activity. One can hope that the efforts of the researchers working to understand this pathology will soon lead to progress in the treatment of these lymphomas.

\section{Acknowledgment}

The authors are thankful to Paul Coppo and Aurélien de Reyniès for their collaborative implication in the authors' work on NKTCL.

\section{References}

[1] S. Swerdlow, E. Campo, N. Harris, E. Jaffe, and S. H. S. Pileri, WHO Classification of Tumors of the Haematopoietic and Lymphoid Tissues, International Agency of Research on Cancer (IARC), Lyon, France, 2008.

[2] W. Y. Au, D. D. Weisenburger, T. Intragumtornchai et al., "Clinical differences between nasal and extranasal natural killer/T-cell lymphoma: a study of 136 cases from the International Peripheral T-Cell Lymphoma Project," Blood, vol. 113, no. 17, pp. 3931-3937, 2009.

[3] J. K. C. Chan, V. C. Sin, K. F. Wong et al., "Nonnasal lymphoma expressing the natural killer cell marker CD56: 
a clinicopathologic study of 49 cases of an uncommon aggressive neoplasm," Blood, vol. 89, no. 12, pp. 4501-4513, 1997.

[4] J. Lee, H. P. Yeon, S. K. Won et al., "Extranodal nasal type NK/T-cell Lymphoma: elucidating clinical prognostic factors for risk-based stratification of therapy," European Journal of Cancer, vol. 41, no. 10, pp. 1402-1408, 2005.

[5] E. Takahashi, N. Asano, C. Li et al., "Nodal T/NK-cell lymphoma of nasal type: a clinicopathological study of six cases," Histopathology, vol. 52, no. 5, pp. 585-596, 2008.

[6] A. Aviles, L. Rodriguez, R. Guzman, A. Talavera, E. L. Garcia, and J. C. Diaz-Maqueo, "Angiocentric T-cell lymphoma of the nose, paranasal sinuses and hard palate," Hematological Oncology, vol. 10, no. 3-4, pp. 141-147, 1992.

[7] J. K. C. Chan, C. S. Ng, P. K. Hui, S. T. H. Lo, and W. H. Lau, "Angiocentric T-cell lymphoma of the skin: an aggressive lymphoma distinct from mycosis fungoides," American Journal of Surgical Pathology, vol. 12, no. 11, pp. 861-876, 1988.

[8] Y. Ishii, N. Yamanaka, and K. Ogawa, "Nasal T-cell lymphoma as a type of so-called "lethal midline granuloma"' Cancer, vol. 50, no. 11, pp. 2336-2344, 1982.

[9] N. L. Harris, E. S. Jaffe, H. Stein et al., "A revised EuropeanAmerican classification of lymphoid neoplasms: a proposal from the International Lymphoma Study Group," Blood, vol. 84, no. 5, pp. 1361-1392, 1994.

[10] J. F. Emile, M. L. Boulland, C. Haioun et al., "CD5-CD56+ Tcell receptor silent peripheral T-cell lymphomas are natural killer cell lymphomas," Blood, vol. 87, no. 4, pp. 1466-1473, 1996.

[11] J. Suzumiya, M. Takeshita, N. Kimura et al., "Expression of adult and fetal natural killer cell markers in sinonasal lymphomas," Blood, vol. 83, no. 8, pp. 2255-2260, 1994.

[12] P. Kanavaros, M. C. Lescs, J. Briere et al., "Nasal Tcell lymphoma: a clinicopathologic entity associated with peculiar phenotype and with Epstein-Barr virus," Blood, vol. 81, no. 10, pp. 2688-2695, 1993.

[13] A. K. Ruskova, R. Thula, and G. T. C. Chan, "Aggressive natural killer-cell leukemia: report of five cases and review of the literature," Leukemia and Lymphoma, vol. 45, no. 12, pp. 2427-2438, 2004.

[14] A. K. S. Chiang, K. Y. Wong, A. C. T. Liang, and G. Srivastava, "Comparative analysis of Epstein-Barr virus gene polymorphisms in nasal T/NK-cell lymphomas and normal nasal tissues: implications on virus strain selection in malignancy," International Journal of Cancer, vol. 80, no. 3, pp. 356-364, 1999.

[15] J. Minarovits, L. F. Hu, S. Imai et al., "Clonality, expression and methylation patterns of the Epstein-Barr virus genomes in lethal midline granulomas classified as peripheral angiocentric T cell lymphomas," Journal of General Virology, vol. 75, no. 1, pp. 77-84, 1994.

[16] A. Jaccard, N. Gachard, B. Marin et al., "Efficacy of L-asparaginase with methotrexate and dexamethasone (AspaMetDex regimen) in patients with refractory or relapsing extranodal NK/T-cell lymphoma, a phase 2 study," Blood, vol. 117, no. 6, pp. 1834-1839, 2011.

[17] J. R. Anderson, J. O. Armitage, and D. D. Weisenburger, "Epidemiology of the non-Hodgkin's lymphomas: distributions of the major subtypes differ by geographic locations," Annals of Oncology, vol. 9, no. 7, pp. 717-720, 1998.

[18] H. Kohrt and R. Advani, "Extranodal natural killer/Tcell lymphoma: current concepts in biology and treatment biology and treatment," Leukemia and Lymphoma, vol. 50, no. 11, pp. 1773-1784, 2009.

[19] J. M. Vose, M. Neumann, and M. E. Harris, "International peripheral T-cell and natural killer/T-cell lymphoma study: pathology findings and clinical outcomes international T-cell lymphoma project," Journal of Clinical Oncology, vol. 26, no. 25, pp. 4124-4130, 2008.

[20] C. Bossard, K. Belhadj, F. Reyes et al., "Expression of the granzyme B inhibitor PI9 predicts outcome in nasal NK/Tcell lymphoma: results of a Western series of 48 patients treated with first-line polychemotherapy within the Groupe d'Etude des Lymphomes de l'Adulte (GELA) trials," Blood, vol. 109, no. 5, pp. 2183-2189, 2007.

[21] T. M. Kim, S. Y. Lee, Y. K. Jeon et al., "Clinical heterogeneity of extranodal NK/T-cell lymphoma, nasal type: a national survey of the Korean Cancer Study Group," Annals of Oncology, vol. 19, no. 8, pp. 1477-1484, 2008.

[22] W. Yong, W. Zheng, J. Zhu et al., "L-asparaginase in the treatment of refractory and relapsed extranodal NK/ T-cell lymphoma, nasal type," Annals of Hematology, vol. 88, no. 7, pp. 647-652, 2009.

[23] V. E. Reyes Jr., T. Al-Saleem, V. G. Robu, and M. R. Smith, "Extranodal NK/T-cell lymphoma nasal type: efficacy of pegaspargase. Report of two patients from the United Sates and review of literature," Leukemia Research, vol. 34, no. 1, pp. e50-e54, 2010.

[24] Y. Huang, A. De Reyniès, L. De Leval et al., "Gene expression profiling identifies emerging oncogenic pathways operating in extranodal NK/T-cell lymphoma, nasal type," Blood, vol. 115, no. 6, pp. 1226-1237, 2010.

[25] J. Iqbal, D. D. Weisenburger, A. Chowdhury et al., "Natural killer cell lymphoma shares strikingly similar molecular features with a group of non-hepatosplenic $\gamma \delta$ T-cell lymphoma and is highly sensitive to a novel aurora kinase A inhibitor in vitro," Leukemia, vol. 25, no. 2, pp. 348-358, 2011.

[26] T. Nagato, H. Kobayashi, K. Kishibe et al., "Expression of interleukin-9 in nasal natural killer/T-cell lymphoma cell lines and patients," Clinical Cancer Research, vol. 11, no. 23, pp. 8250-8257, 2005.

[27] Y. Nakashima, H. Tagawa, R. Suzuki et al., "Genome-wide array-based comparative genomic hybridization of natural killer cell lymphoma/leukemia: different genomic alteration patterns of aggressive NK-cell leukemia and extranodal NK/T-cell lymphoma, nasal type," Genes Chromosomes and Cancer, vol. 44, no. 3, pp. 247-255, 2005.

[28] S.-B. Ng, V. Selvarajan, G. Huang et al., "Activated oncogenic pathways and therapeutic targets in extranodal nasal-type NK/T cell lymphoma revealed by gene expression profiling," Journal of Pathology, vol. 223, no. 4, pp. 496-510, 2011.

[29] T. Oka, T. Yoshino, K. Hayashi et al., "Reduction of hematopoietic cell-specific tyrosine phosphatase SHP-1 gene expression in natural killer cell lymphoma and various types of lymphomas/leukemias: combination analysis with cDNA expression array and tissue microarray," American Journal of Pathology, vol. 159, no. 4, pp. 1495-1505, 2001.

[30] Y. Zhang, J. H. Ohyashiki, T. Takaku, N. Shimizu, and K. Ohyashiki, "Transcriptional profiling of Epstein-Barr virus (EBV) genes and host cellular genes in nasal NK/T-cell lymphoma and chronic active EBV infection," British Journal of Cancer, vol. 94, no. 4, pp. 599-608, 2006.

[31] H. E. Heslop, K. S. Slobod, M. A. Pule et al., "Longterm outcome of EBV-specific T-cell infusions to prevent or treat EBV-related lymphoproliferative disease in transplant recipients," Blood, vol. 115, no. 5, pp. 925-935, 2010. 
[32] A. K. S. Chiang, Q. Tao, G. Srivastava, and F. C. S. Ho, "Nasal NK- and T-cell lymphomas share the same type of Epstein-Barr virus latency as nasopharyngeal carcinoma and Hodgkin's disease," International Journal of Cancer, vol. 68, no. 3, pp. 285-290, 1996.

[33] C. M. Bollard, S. Gottschalk, A. M. Leen et al., "Complete responses of relapsed lymphoma following genetic modification of tumor-antigen presenting cells and T-lymphocyte transfer," Blood, vol. 110, no. 8, pp. 2838-2845, 2007.

[34] C. P. Fox, T. A. Haigh, G. S. Taylor et al., "A novel latent membrane 2 transcript expressed in Epstein-Barr viruspositive NK- and T-cell lymphoproliferative disease encodes a target for cellular immunotherapy," Blood, vol. 116, no. 19, pp. 3695-3704, 2010.

[35] T. Kaneko, J. Fukuda, T. Yoshihara et al., "Nasal natural killer (NK) cell lymphoma: report of a case with activated NK cells containing Epstein-Barr virus and expressing CD21 antigen, and comparative studies of their phenotype and cytotoxicity with normal NK cells," British Journal of Haematology, vol. 91, no. 2, pp. 355-361, 1995.

[36] J. Tabiasco, A. Vercellone, F. Meggetto, D. Hudrisier, P. Brousset, and J. J. Fournié, "Acquisition of viral receptor by NK cells through immunological synapse," Journal of Immunology, vol. 170, no. 12, pp. 5993-5998, 2003.

[37] H. Kimura, Y. Hoshino, H. Kanegane et al., "Clinical and virologic characteristics of chronic active Epstein-Barr virus infection," Blood, vol. 98, no. 2, pp. 280-286, 2001.

[38] S. E. Straus, "The chronic mononucleosis syndrome," Journal of Infectious Diseases, vol. 157, no. 3, pp. 405-412, 1988.

[39] J. I. Cohen, H. Kimura, S. Nakamura, Y. H. Ko, and E. S. Jaffe, "Epstein-Barr virus-associated lymphoproliferative disease in non-immunocompromised hosts: a status report and summary of an international meeting, 8-9 September 2008," Annals of Oncology, vol. 20, no. 9, pp. 1472-1482, 2009.

[40] M. L. Boulland, V. Meignin, K. Leroy-Viard et al., "Human interleukin-10 expression in T/natural killer-cell lymphomas: association with anaplastic large cell lymphomas and nasal natural killer- cell lymphomas," American Journal of Pathology, vol. 153, no. 4, pp. 1229-1237, 1998.

[41] J. Teruya-Feldstein, E. S. Jaffe, P. R. Burd et al., "The role of Mig, the monokine induced by interferon- $\gamma$, and IP-10, the interferon- $\gamma$-inducible protein-10, in tissue necrosis and vascular damage associated with Epstein-Barr virus-positive lymphoproliferative disease," Blood, vol. 90, no. 10, pp. 40994105, 1997.

[42] P. Kanavaros, M. L. Boulland, B. Petit, B. Arnulf, and P. Gaulard, "Expression of cytotoxic proteins in peripheral Tcell and natural killer-cell (NK) lymphomas: association with extranodal site, NK or $\mathrm{T} \gamma \delta$ phenotype, anaplastic morphology and CD30 expression," Leukemia and Lymphoma, vol. 38, no. 3-4, pp. 317-326, 2000.

[43] E. Vivier, E. Tomasello, M. Baratin, T. Walzer, and S. Ugolini, "Functions of natural killer cells," Nature Immunology, vol. 9, no. 5, pp. 503-510, 2008.

[44] C. Schmitt, B. Ghazi, and A. Bensussan, "NK cells and surveillance in humans," Reproductive BioMedicine Online, vol. 16, no. 2, pp. 192-201, 2008.

[45] P. Parham, "MHC class I molecules and KIRS in human history, health and survival," Nature Reviews Immunology, vol. 5, no. 3, pp. 201-214, 2005.

[46] Y. Suto, K. Maenaka, T. Yabe et al., "Chromosomal localization of the human natural killer cell class I receptor family genes to 19q13.4 by fluorescence in situ hybridization," Genomics, vol. 35, no. 1, pp. 270-272, 1996.
[47] S. I. Khakoo and M. Carrington, "KIR and disease: a model system or system of models?" Immunological Reviews, vol. 214, no. 1, pp. 186-201, 2006.

[48] W. Haedicke, F. C. S. Ho, A. Chott et al., "Expression of CD94/NKG2A and killer immunoglobulin-like receptors in NK cells and a subset of extranodal cytotoxic T-cell lymphomas," Blood, vol. 95, no. 11, pp. 3628-3630, 2000.

[49] C. W. Lin, W. H. Lee, C. L. Chang, J. Y. Yang, and S. M. $\mathrm{Hsu}$, "Restricted killer cell immunoglobulin-like receptor repertoire without T-cell receptor $\gamma$ rearrangement supports a true natural killer-cell lineage in a subset of sinonasal lymphomas," American Journal of Pathology, vol. 159, no. 5, pp. 1671-1679, 2001.

[50] R. Lundell, L. Hartung, S. Hill, S. L. Perkins, and D. W. Bahler, "T-cell large granular lymphocyte leukemias have multiple phenotypic abnormalities involving pan-Tcell antigens and receptors for MHC molecules," American Journal of Clinical Pathology, vol. 124, no. 6, pp. 937-946, 2005.

[51] A. Dalloul, L. Laroche, M. Bagot et al., "Interleukin-7 is a growth factor for Sezary lymphoma cells," Journal of Clinical Investigation, vol. 90, no. 3, pp. 1054-1060, 1992.

[52] N. Ortonne, S. Le Gouvello, H. Mansour et al., "CD158K/KIR3DL2 transcript detection in lesional skin of patients with erythroderma is a tool for the diagnosis of Sézary syndrome," Journal of Investigative Dermatology, vol. 128, no. 2, pp. 465-472, 2008.

[53] E. Poszepczynska-Guigné, V. Schiavon, M. D’Incan et al., "CD158k/KIR3DL2 is a new phenotypic marker of sezary cells: relevance for the diagnosis and follow-up of sezary syndrome," Journal of Investigative Dermatology, vol. 122, no. 3, pp. 820-823, 2004.

[54] F. Takei, K. L. McQueen, M. Maeda et al., "Ly49 and CD94/NKG2: developmentally regulated expression and evolution," Immunological Reviews, vol. 181, pp. 90-103, 2001.

[55] C. W. Lin, Y. H. Chen, Y. C. Chuang, T. Y. Liu, and S. M. Hsu, "CD94 transcripts imply a better prognosis in nasaltype extranodal NK/T-cell lymphoma," Blood, vol. 102, no. 7, pp. 2623-2631, 2003.

[56] L. A. Fernandez, B. Pope, C. Lee, and E. Zayed, "Aggressive natural killer cell leukemia in an adult with establishment of an NK cell line," Blood, vol. 67, no. 4, pp. 925-930, 1986.

[57] S. Koizumi, H. Seki, and T. Tachinami, "Malignant clonal expansion of large granular lymphocytes with a Leu-11+, Leu-7- surface phenotype: in vitro responsiveness of malignant cells to recombinant human interleukin 2," Blood, vol. 86, no. 5, pp. 1065-1073, 1986.

[58] C. J. Froelich, V. M. Dixit, and X. Yang, "Lymphocyte granule-mediated apoptosis: matters of viral mimicry and deadly proteases," Immunology Today, vol. 19, no. 1, pp. 3036, 1998.

[59] M. B. Barrie, H. W. Stout, M. S. Abougergi, B. C. Miller, and D. L. Thiele, "Antiviral cytokines induce hepatic expression of the granzyme B inhibitors, proteinase inhibitor 9 and serine proteinase inhibitor 6," Journal of Immunology, vol. 172, no. 10, pp. 6453-6459, 2004.

[60] B. A. Bladergroen, C. J. L. M. Meijer, R. L. Ten Berge et al., "Expression of the granzyme B inhibitor, protease inhibitor 9, by tumor cells in patients with non-Hodgkin and Hodgkin lymphoma: a novel protective mechanism for tumor cells to circumvent the immune system?” Blood, vol. 99, no. 1, pp. 232-237, 2002.

[61] C. S. Ng, S. T. H. Lo, J. K. C. Chan, and W. C. Chan, "CD56+ putative natural killer cell lymphomas: production 
of cytolytic effectors and related proteins mediating tumor cell apoptosis?” Human Pathology, vol. 28, no. 11, pp. 1276$1282,1997$.

[62] K. Aozasa, T. Takakuwa, T. Hongyo, and W. I. Yang, "Nasal NK/T-cell lymphoma: epidemiology and pathogenesis," International Journal of Hematology, vol. 87, no. 2, pp. 110-117, 2008.

[63] T. Takakuwa, Z. Dong, S. Nakatsuka et al., "Frequent mutations of Fas gene in nasal NK/T cell lymphoma," Oncogene, vol. 21, no. 30, pp. 4702-4705, 2002.

[64] M. Yamaguchi, K. Kita, H. Miwa et al., "Frequent expression of P-glycoprotein/MDR1 by nasal T-cell lymphoma cells," Cancer, vol. 76, no. 11, pp. 2351-2356, 1995.

[65] Y. H. Ko, H. J. Ree, W. S. Kim, W. H. Choi, W. S. Moon, and S. W. Kim, "Clinicopathologic and genotypic study of extranodal nasal-type natural killer/T-cell lymphoma and natural killer precursor lymphoma among Koreans," Cancer, vol. 89, no. 10, pp. 2106-2116, 2000.

[66] Y. H. Ko, K. E. Choi, J. H. Han, J. M. Kim, and H. J. Ree, "Comparative genomic hybridization study of nasaltype NK/T-cell lymphoma," Communications in Clinical Cytometry, vol. 46, no. 2, pp. 85-91, 2001.

[67] L. L. Siu, V. Chan, J. K. C. Chan, K. F. Wong, R. Liang, and Y. L. Kwong, "Consistent patterns of allelic loss in natural killer cell lymphoma," American Journal of Pathology, vol. 157, no. 6, pp. 1803-1809, 2000.

[68] L. L. Siu, K. F. Wong, J. K. C. Chan, and Y. L. Kwong, "Comparative genomic hybridization analysis of natural killer cell lymphoma/leukemia: recognition of consistent patterns of genetic alterations," American Journal of Pathology, vol. 155, no. 5, pp. 1419-1425, 1999.

[69] H. S. Sun, I. J. Su, Y. C. Lin, J. S. Chen, and S. Y. Fang, "A $2.6 \mathrm{Mb}$ interval on chromosome 6q25.2-q25.3 is commonly deleted in human nasal natural killer/T-cell lymphoma," British Journal of Haematology, vol. 122, no. 4, pp. 590-599, 2003.

[70] J. Yoon and Y. H. Ko, "Deletion mapping of the long arm of chromosome 6 in peripheral T and NK cell lymphomas," Leukemia and Lymphoma, vol. 44, no. 12, pp. 2077-2082, 2003.

[71] J. Iqbal, C. Kucuk, R. J. deLeeuw et al., "Genomic analyses reveal global functional alterations that promote tumor growth and novel tumor suppressor genes in natural killercell malignancies," Leukemia, vol. 23, no. 6, pp. 1139-1151, 2009.

[72] A. Kuma, M. Hatano, M. Matsui et al., "The role of autophagy during the early neonatal starvation period," Nature, vol. 432, no. 7020, pp. 1032-1036, 2004.

[73] M. M. Hippert, P. S. O’Toole, and A. Thorburn, "Autophagy in cancer: good, bad, or both?” Cancer Research, vol. 66, no. 19, pp. 9349-9351, 2006.

[74] M. E. Ray, G. Wistow, Y. A. Su, P. S. Meltzer, and J. M. Trent, "AIM1, a novel non-lens member of the $\beta \gamma$-crystallin superfamily, is associated with the control of tumorigenicity in human malignant melanoma," Proceedings of the National Academy of Sciences of the United States of America, vol. 94, no. 7, pp. 3229-3234, 1997.

[75] G. A. Martins, L. Cimmino, M. Shapiro-Shelef et al., "Transcriptional repressor Blimp-1 regulates $\mathrm{T}$ cell homeostasis and function," Nature Immunology, vol. 7, no. 5, pp. 457-465, 2006.

[76] C. A. Turner Jr., D. H. Mack, and M. M. Davis, "Blimp1 , a novel zinc finger-containing protein that can drive the maturation of B lymphocytes into immunoglobulinsecreting cells," Cell, vol. 77, no. 2, pp. 297-306, 1994.

[77] L. Pasqualucci, M. Compagno, J. Houldsworth et al., "Inactivation of the PRDM1/BLIMP1 gene in diffuse large B cell lymphoma," Journal of Experimental Medicine, vol. 203, no. 2, pp. 311-317, 2006.

[78] G. L. Semenza, "Hif-1 and human disease: one highly involved factor," Genes and Development, vol. 14, no. 16, pp. 1983-1991, 2000.

[79] P. J. Jost and J. Ruland, "Aberrant NF- $\kappa$ B signaling in lymphoma: mechanisms, consequences, and therapeutic implications," Blood, vol. 109, no. 7, pp. 2700-2707, 2007.

[80] J. E. Darnell Jr., I. M. Kerr, and G. R. Stark, "Jak-STAT pathways and transcriptional activation in response to IFNs and other extracellular signaling proteins," Science, vol. 264, no. 5164, pp. 1415-1421, 1994.

[81] Q. Zhang, P. N. Raghunath, L. Xue et al., "Multilevel dysregulation of STAT3 activation in anaplastic lymphoma kinasepositive T/null-cell lymphoma," Journal of Immunology, vol. 168, no. 1, pp. 466-474, 2002.

[82] P. Coppo, V. Gouilleux-Gruart, Y. Huang et al., "STAT3 transcription factor is constitutively activated and is oncogenic in nasal-type NK/T-cell lymphoma," Leukemia, vol. 23, no. 9, pp. 1667-1678, 2009.

[83] P. P. Piccaluga, C. Agostinelli, A. Califano et al., "Gene expression analysis of peripheral $\mathrm{T}$ cell lymphoma, unspecified, reveals distinct profiles and new potential therapeutic targets," Journal of Clinical Investigation, vol. 117, no. 3, pp. 823-834, 2007.

[84] T. Wang, G. Niu, M. Kortylewski et al., "Regulation of the innate and adaptive immune responses by Stat-3 signaling in tumor cells," Nature Medicine, vol. 10, no. 1, pp. 48-54, 2004.

[85] D. Rotin and S. Kumar, "Physiological functions of the HECT family of ubiquitin ligases," Nature Reviews Molecular Cell Biology, vol. 10, no. 6, pp. 398-409, 2009.

[86] R. Schmitz, M. L. Hansmann, V. Bohle et al., “TNFAIP3 (A20) is a tumor suppressor gene in Hodgkin lymphoma and primary mediastinal B cell lymphoma," Journal of Experimental Medicine, vol. 206, no. 5, pp. 981-989, 2009.

[87] L. Quintanilla-Martinez, M. Kremer, G. Keller et al., "p53 mutations in nasal natural killer/t-cell lymphoma from mexico: association with large cell morphology and advanced disease," American Journal of Pathology, vol. 159, no. 6, pp. 2095-2105, 2001.

[88] M. Li, D. Chen, A. Shiloh et al., "Deubiquitination of p53 by HAUSP is an important pathway for p53 stabilization," Nature, vol. 416, no. 6881, pp. 648-653, 2002.

[89] M. Kimura, Y. Matsuda, T. Eki et al., "Assignment of STK6 to human chromosome $20 \mathrm{q} 13.2 \rightarrow \mathrm{q} 13.1$ and a pseudogene STK6P to 1q41 $\rightarrow$ q42," Cytogenetics and Cell Genetics, vol. 79, no. 3-4, pp. 201-203, 1997.

[90] L. Zhang, M. S. Anglesio, M. O'Sullivan et al., “The E3 ligase HACE1 is a critical chromosome 6q21 tumor suppressor involved in multiple cancers," Nature Medicine, vol. 13, no. 9, pp. 1060-1069, 2007.

[91] M. S. Anglesio, V. Evdokimova, N. Melnyk et al., "Differential expression of a novel ankyrin containing E3 ubiquitinprotein ligase, Hace1, in sporadic Wilms' tumor versus normal kidney," Human Molecular Genetics, vol. 13, no. 18, pp. 2061-2074, 2004.

[92] J. Zhao, Z. Zhang, Z. Vucetic, K. J. Soprano, and D. R. Soprano, "HACE1: a novel repressor of RAR transcriptional activity," Journal of Cellular Biochemistry, vol. 107, no. 3, pp. 482-493, 2009. 
[93] T. Bowman, R. Garcia, J. Turkson, and R. Jove, "STATs in oncogenesis," Oncogene, vol. 19, no. 21, pp. 2474-2488, 2000.

[94] J. F. Bromberg, M. H. Wrzeszczynska, G. Devgan et al., "Stat3 as an oncogene," Cell, vol. 98, no. 3, pp. 295-303, 1999.

[95] R. Chiarle, W. J. Simmons, H. Cai et al., "Stat3 is required for ALK-mediated lymphomagenesis and provides a possible therapeutic target," Nature Medicine, vol. 11, no. 6, pp. 623629, 2005.

[96] A. Zamo, R. Chiarle, R. Piva et al., "Anaplastic lymphoma kinase (ALK) activates Stat3 and protects hematopoietic cells from cell death," Oncogene, vol. 21, no. 7, pp. 1038-1047, 2002.

[97] H. Yu, M. Kortylewski, and D. Pardoll, "Crosstalk between cancer and immune cells: role of STAT3 in the tumour microenvironment," Nature Reviews Immunology, vol. 7, no. 1, pp. 41-51, 2007.

[98] M. Kortylewski, M. Kujawski, T. Wang et al., "Inhibiting Stat3 signaling in the hematopoietic system elicits multicomponent antitumor immunity," Nature Medicine, vol. 11, no. 12, pp. 1314-1321, 2005.

[99] C. Zou, J. Ma, X. Wang et al., "Lack of Fas antagonism by Met in human fatty liver disease," Nature Medicine, vol. 13, no. 9, pp. 1078-1085, 2007.

[100] B. K. Hadland, N. R. Manley, D. M. Su et al., " $\gamma$-secretase inhibitors repress thymocyte development," Proceedings of the National Academy of Sciences of the United States of America, vol. 98, no. 13, pp. 7487-7491, 2001.

[101] T. Palomero, M. L. Sulis, M. Cortina et al., "Mutational loss of PTEN induces resistance to NOTCH1 inhibition in T-cell leukemia," Nature Medicine, vol. 13, no. 10, pp. 1203-1210, 2007.

[102] E. Chanudet, H. Ye, J. Ferry et al., "A20 deletion is associated with copy number gain at the TNFAIBIC locus and occurs preferentially in translocation-negative MALT lymphoma of the ocular adnexa and salivary glands," Journal of Pathology, vol. 217, no. 3, pp. 420-430, 2009.

[103] L. L. Siu, J. K. C. Chan, K. F. Wong, and Y. L. Kwong, "Specific patterns of gene methylation in natural killer cell lymphomas: p73 is consistently involved," American Journal of Pathology, vol. 160, no. 1, pp. 59-66, 2002.

[104] V. Rouget-Quermalet, J. Giustiniani, A. Marie-Cardine et al., "Protocadherin 15 (PCDH15): a new secreted isoform and a potential marker for NK/T cell lymphomas," Oncogene, vol. 25, no. 19, pp. 2807-2811, 2006.

[105] M. Ito, T. Maruyama, N. Saito, S. Koganei, K. Yamamoto, and N. Matsumoto, "Killer cell lectin-like receptor G1 binds three members of the classical cadherin family to inhibit NK cell cytotoxicity," Journal of Experimental Medicine, vol. 203, no. 2, pp. 289-295, 2006.

[106] J. R. Bischoff and G. D. Plowman, "The Aurora/Ipllp kinase family: regulators of chromosome segregation and cytokinesis," Trends in Cell Biology, vol. 9, no. 11, pp. 454459, 1999.

[107] H. Katayama, K. Sasai, H. Kawai et al., "Phosphorylation by aurora kinase A induces Mdm2-mediated destabilization and inhibition of p53," Nature Genetics, vol. 36, no. 1, pp. 55-62, 2004.

[108] H. G. Drexler and Y. Matsuo, "Malignant hematopoietic cell lines: in vitro models for the study of natural killer cell leukemia-lymphoma," Leukemia, vol. 14, no. 5, pp. 777-782, 2000.

[109] S. Zhao, Q. L. Tang, M. X. He et al., "A novel nude mice model of human extranodal nasal type NK/T-cell lymphoma," Leukemia, vol. 22, no. 1, pp. 170-178, 2008. 


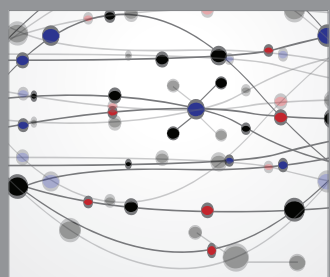

The Scientific World Journal
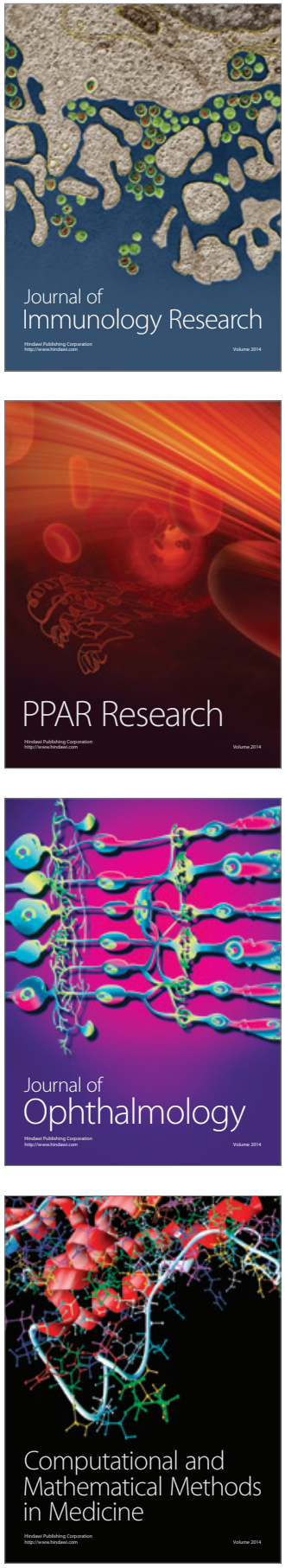

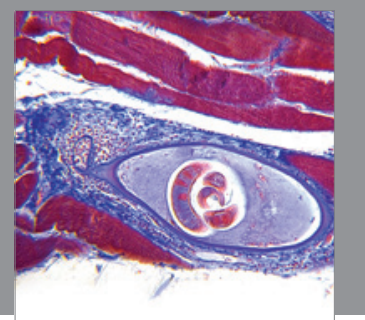

Gastroenterology

Research and Practice
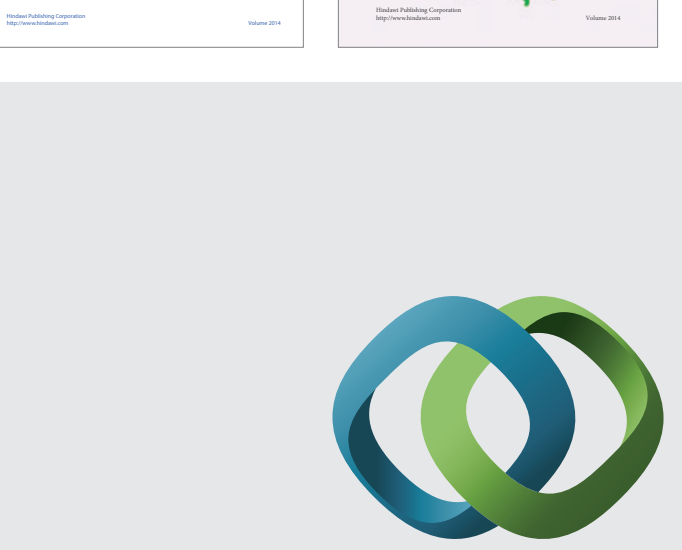

\section{Hindawi}

Submit your manuscripts at

http://www.hindawi.com
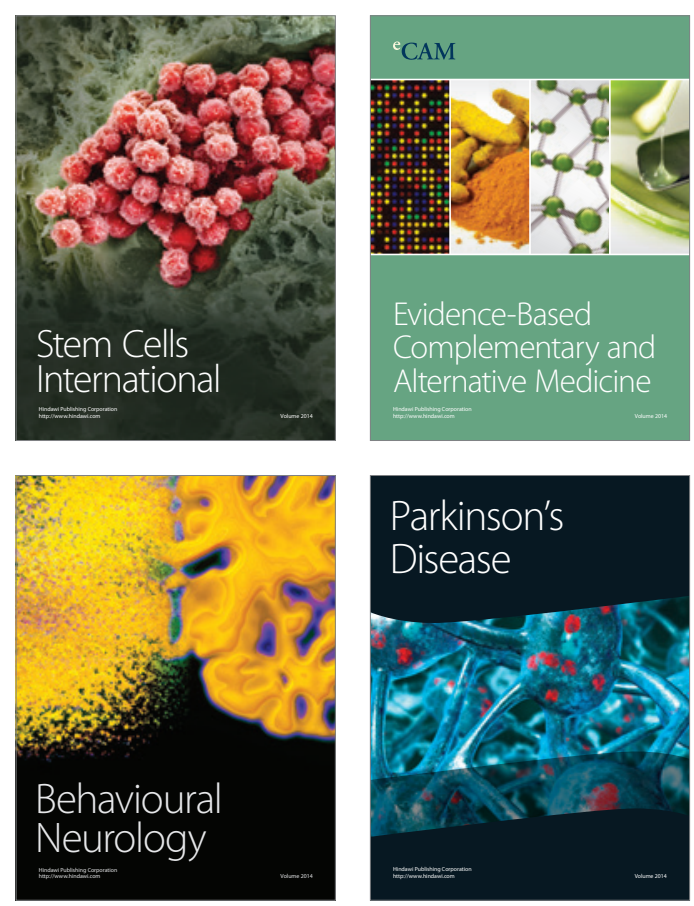

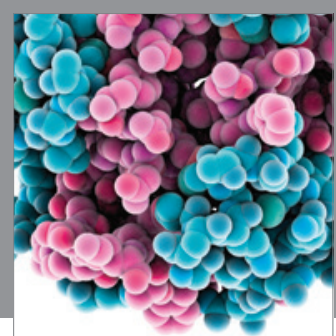

Journal of
Diabetes Research

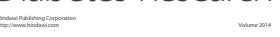

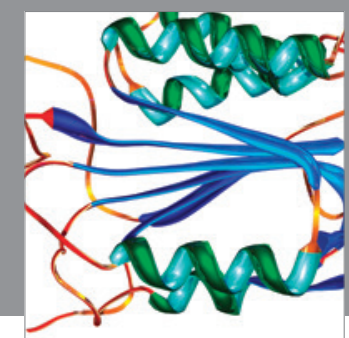

Disease Markers
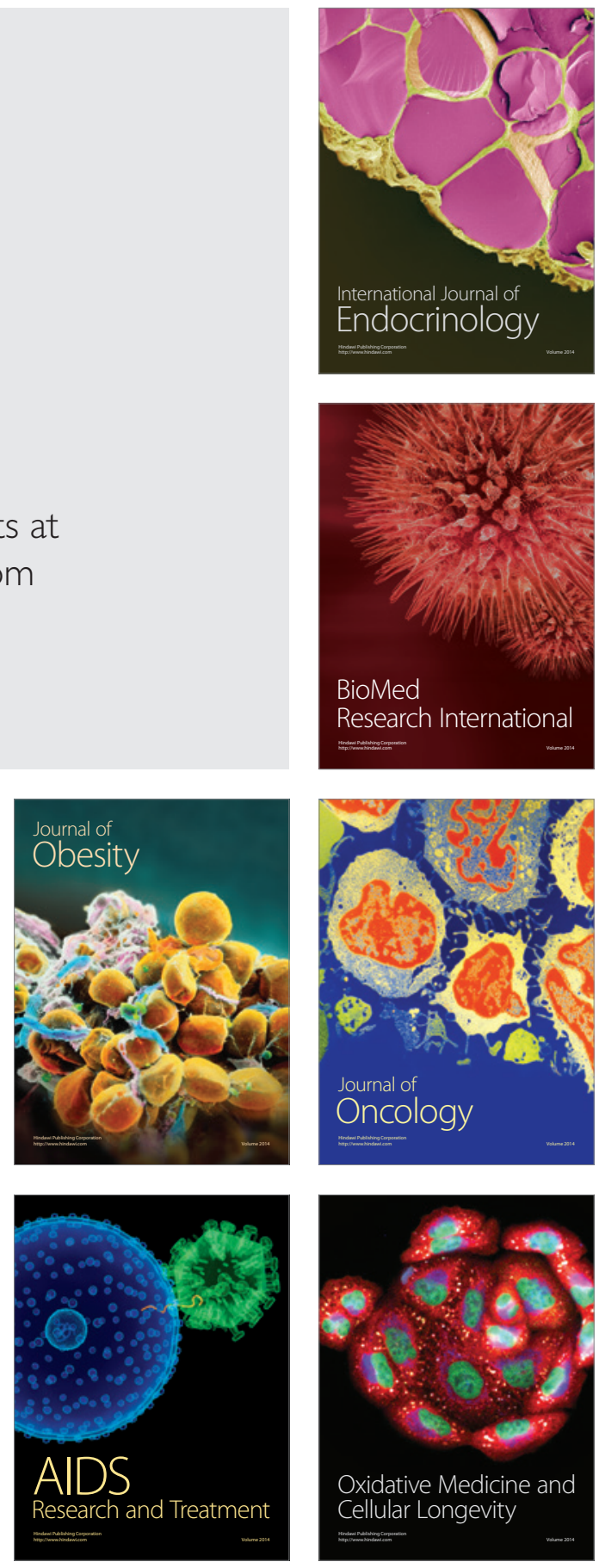